\title{
The Hierarchy Structure in Directed and Undirected Signed Networks
}

\author{
Jamal Maktoubian $^{1^{*}}$, Mohebollah Noori², Mahta Amini ${ }^{3}$, Mehran Ghasempour-Mouziraji ${ }^{4}$ \\ ${ }^{1}$ International School of Information Management (ISIM), University of Mysore, Mysore, India \\ ${ }^{2}$ Zarghan Branch, Islamic Azad University, Zarghan, Iran \\ ${ }^{3}$ Department of Computer Science, Shahid Beheshti University, Tehran, Iran \\ ${ }^{4}$ Department of Engineering, Islamic Azad University of Sari, Sari, Iran \\ Email: `Jamal@isim.net.in, Nooriweb21@gmail.com, mahta_amini7@yahoo.com,mehran_ghasempour20@yahoo.com
}

How to cite this paper: Maktoubian, J., Noori, M., Amini, M. and GhasempourMouziraji, M. (2017) The Hierarchy Structure in Directed and Undirected Signed Networks. Int. J. Communications, Network and System Sciences, 10, 209-222.

https://doi.org/10.4236/ijcns.2017.1010012

Received: August 30, 2017

Accepted: October 8, 2017

Published: October 11, 2017

Copyright $\odot 2017$ by authors and Scientific Research Publishing Inc. This work is licensed under the Creative Commons Attribution International License (CC BY 4.0).

http://creativecommons.org/licenses/by/4.0/

\begin{abstract}
The concept of social stratification and hierarchy among human dates is back to the origin of human race. Presently, the growing reputation of social networks has given us with an opportunity to analyze these well-studied phenomena over different networks at different scales. Generally, a social network could be defined as a collection of actors and their interactions. In this work, we concern ourselves with a particular type of social networks, known as trust networks. In this type of networks, there is an explicit show of trust (positive interaction) or distrust (negative interaction) among the actors. In a social network, actors tend to connect with each other on the basis of their perceived social hierarchy. The emergence of such a hierarchy within a social community shows the manner in which authority manifests in the community. In the case of signed networks, the concept of social hierarchy can be interpreted as the emergence of a tree-like structure comprising of actors in a top-down fashion in the order of their ranks, describing a specific parent-child relationship, viz. child trusts parent. However, owing to the presence of positive as well as negative interactions in signed networks, deriving such "trust hierarchies" is a non-trivial challenge. We argue that traditional notions (of unsigned networks) are insufficient to derive hierarchies that are latent within signed networks.
\end{abstract}

\section{Keywords}

Unsigned Network, Signed Network, Global Reaching Centrality (GRC), Hierarchy, Social Network 


\section{Introduction}

Structural analysis of complex networks has been a dynamic and challenging area of interest among researchers for the past few decades [1]. In a generic sense, a network is a collection of nodes associated to the other through links [2]. Several graph theoretic approaches over such networks have revealed certain fundamental facts. Evidently, network analysis could provide us with better insights in understanding the hidden aspects of individuals or groups involved within a network, the pattern of relationships, how they evolve etc [3]. Any network could be represented as a graph consisting of a collection of nodes (units) and edges (interactions) [4]. In a network, the manner in which one node interacts with the other displays an important feature, the connectedness among nodes. The nature of connectedness underlying a network also determines its complex topology. In other words, network complexity is an intrinsic property of any physical, chemical, biological or social system characterized by various nodes and their interactions [5]. Examples include organizational networks, neural networks, protein interaction networks, Internet, the World Wide Web and social networks to name but a few.

The past decade witnessed a tremendous rise in the popularity of online social networks such as Twitter, Digg, Youtube, Delicious, Livejournal, Facebook etc. Our study mainly focuses on the analyses of similar online social networks in order to understand the underlying mechanism of the connections involved as well as to verify the existence of certain social phenomena within the networks. Broadly speaking, a social network could be directed or undirected depending on the type of edges present in them. Directed social networks are distinguished from undirected ones by the presence of directed edges between actors [6]. An example (Figure 1) for directed network could be followership in Twitter where an actor simply 'follows' another. Alternatively, undirected social networks comprise of undirected edges between actors. Facebook is an example for undirected networks with edges depicting only mutual friendships.

Another type of classification termed as the trust networks deals with nature of interactions (positive or negative) involved in social networks. In this type of classification, a social network could be categorized as either signed or unsigned. Unsigned networks are described by the presence of a single type of interaction, usually being positive in nature. That is, in unsigned networks all actors are same, either friends or strangers. Generally, social networks are largely found to be unsigned in nature [7]. Followership in Twitter and friendship in Facebook

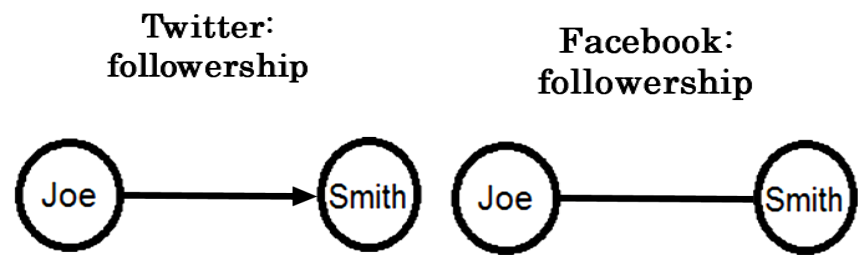

Figure 1. Examples of directed and undirected network connectivity. 
are typical examples. But in the real world, the relationships need not always be positive in nature. Signed networks, capture this aspect of society allowing explicit show of trust or distrust among actors. They can designate others as friends or foes [8]. In this scenario, an actor is said to trust the other if an actor approves of one's opinion among themselves. At the same time, an actor is said to distrust the other if an actor disapproves of one's opinion. E-opinions, Slashdot Zoo network are some of the examples of signed networks that indicate trust/friends or distrust/foes explicitly among themselves using an edge-weight of +1 and -1 respectively. Mathematically, a signed network can be defined as a directed graph, $\mathrm{G}=(\mathrm{V}, \mathrm{E})$ where i) $\mathrm{V}$ is the set of actors in a network, $\mathrm{E} \subseteq \mathrm{V} \times \mathrm{V}$ is the set of edges such that $(\mathrm{u}, \mathrm{v})$ indicates a link between $\mathrm{u} \in \mathrm{V}$ and $\mathrm{v} \in \mathrm{V}$ s: $\mathrm{E} \rightarrow$ $\{+1,-1\}$ assigns the edge weight [9]. Consider the following illustration in Figure 2. If node $A$ is connected to node $B$ as a friend, there should be a directed edge from node $A$ to node $B$ with a trust score of +1 . Meanwhile, if $A$ is connected to $B$ as a foe, there should be an edge directed from A to B with a score of -1 .

\section{Background and Prior Work}

Various aspects of hierarchy have been studied in many literatures till date. The general idea behind the concept of hierarchy can be stated as the emergence of a tree-like structure in a top-down fashion in the order of their ranks further depicting a specific relationship. Earlier studies on dominance relationship in animal societies, Bonabeau et al. suggest a process of self-organization of nodes depending on their roles and importance [10]. This lead to the identification of important or 'leader' nodes within a community. Such nodes occupy the higher positions in the hierarchy. Therefore, it can be argued that in a hierarchy the higher node indicates a greater influence than the lower ones. Using the directional correlation function analysis, M. Nagy et al. found that similar dominance hierarchies exist in the case of pigeon flocks [11].

In 1984, Huseyn et al. [12] suggested that hierarchy is found in numerous complex systems. Hierarchical organization is also studied in different real networks such as actor network, language network, the Internet and World Wide Web by Ravasz and Barabási in 2003 [13]. They proposed that many real networks are scale free and transitive in nature which can be seen as a consequence of the hierarchy underlying the network. Small groups of nodes rearrange themselves to form a hierarchy of larger groups. In order to examine the presence of hierarchical structure in real networks, they argued that the scaling law for the clustering coefficient $\mathrm{Ck}$, is sufficient to quantify the existence of hierarchy of nodes [14].

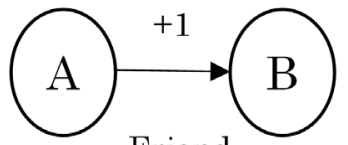

Friend

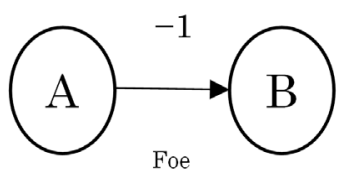

Foe

Figure 2. Examples for signed network connectivity. 
Likewise, hierarchy is observed in certain types of collaboration networks too. Rowe et al. [15], proposed a novel algorithm to find social hierarchy in e-mail networks by introducing a social score S. This score is computed for each user as a weighted combination of several other measures including the number of e-mails exchanged. Several studies came up with different hierarchy measures that lacked universal applicability on all network types. Instead of employing different measures, the need for a single efficient measure for quantifying hierarchy in complex networks was inevitable.

Liben-Nowell and E. Gilbert et al. [16] [17] Studied on social networks dealt with link-prediction and tie-strength prediction. They addressed the link-pre-diction issue and discuss certain achievements based on proximity measures of nodes in a network. Rather than considering the network evolution, a static snapshot of the network along with some specific node attributes are taken into study. Link-prediction can be applied to social network analysis to find out interesting or promising interactions within its members. In 2009, E. Gilbert et al. provided a predictive model for tie strength. The model effectively distinguishes between strong and weak ties with over $85 \%$ accuracy. The model predicts the tie strength by observing the manner in which a user chooses to communicate to another user in particular regardless of the number other choices offered.

Apart from these, attempts have been made lately to explore hierarchies as well. Helic D. and Strohmaier M. [18] looked into usefulness of tag hierarchies in improving navigability in social tagging sites like Delicious, CiteULike and Flickr. This paper aims to explore the usefulness of tag hierarchies as directories to facilitate navigation or browsing in social tagging systems. In order to construct such a tag hierarchy, the authors have put forward a new version of an existing centrality based algorithm with a branching factor $b$ as an input parameter which describes the maximum number of categories and sub-categories. It employs tag co-occurrence as the similarity measure and tag generality as the centrality measure over the tag-tag networks. In a tag network, each tag is considered as a node and is linked to the other node according to a certain occurrence threshold. In the process of building up a hierarchy, the nodes are first ranked in a descending order based on the degree of centrality (generality threshold) to obtain a centrality list. As a result, the most general tags are placed at the top order.

The proposed algorithm has two phase procedure to ensure that as much as the given tags are being connected to the main tree without the tree being fairly deep. In the first phase, it populates a forest of multiple trees with the most general node as the root node, iterates through the centrality list, identifies the most similar tag to the current tag in the tree computing the co-occurrence threshold and then appends the tag as a child to its most similar tags. It attaches a maximum of subcategories to a given category. Later the produced trees are sorted in descending order of their size (no: of categories they possess) and the largest tree is considered as the main tree. In the second phase, the algorithm appends the other trees to the main tree by connecting the root node of a particular tree to 
the most similar node in the main tree. In case the most similar mode consists of only one free sub-category spot, then a misc category is introduced into the free spot and then the given tree is appended to that misc category. However, the nesting of misc category is also necessary and cannot be avoided completely due to the very structure of tag-tag networks. Normally, in a typical power-law network, the nodes with high degree centrality are connected to a small number of high and mid degree nodes (high centrality) as well as to a large number of low degree nodes (low centrality). Such high centrality tags occupy the top positions of the hierarchy. Therefore, in the hierarchy building process, the algorithm first appends the adjacent high degree and the mid degree nodes as sub-categories to a given node using up all free sub-category spots followed by the addition of the low degree nodes through the misc category. It is to be noted that nested misc categories do not affect the semantics of the network but rather keep the tags away from the most related ones into its misc categories. The results and simulation studies illustrates that the proposed algorithm outperforms existing ones in constructing a tag hierarchy useful for better navigation.

Maiya and Berger-Wolf [19] introduced a simple and flexible method based on maximum likelihood to infer social hierarchy from weighted social networks. They have used a simple greedy algorithm to infer maximum likelihood hierarchy from a given network. This approach was evaluated against both simulated as well as real-world datasets for accuracy. This method can also be used to infer the generative interaction models that could lead to a social network. The results show that hierarchies can be inferred from the associations among different entities in a network, provides the frequency and occurrence of theses associations.

Gupte et al. [20] investigated the emergence of hierarchy in directed social networks. They propose a measure of hierarchy and illustrate how hierarchy and degree of stratification emerge with the increase in network size. The paper presents a measure of hierarchy and a polynomial time algorithm to find the largest hierarchy in directed networks. This paper also shows that with the increase in network size, the size of hierarchy grows significantly but the rate of stratification tends to be slow. The studies are based on the assumption that there exists a global social rank for every person in a network and each of them is aware of their ranks as well as the ranks of people they link to. It is been observed that when people of higher ranks in a hierarchy links (or recommends) people of lower ranks there occurs a considerable amount of social agony depending on the difference between their ranks.

\section{Global Reaching Centrality (GRC)}

In 2012, the problem has been examined and a universal hierarchy measure has recently been put forward by Mones et al. [21]. Known as the Global Reaching Centrality (GRC), this new measure captures the heterogeneous distribution of local reaching centralities in a network. Unlike other measures so far suggested, GRC claims to overcome many drawbacks and is widely applicable to all classes 
of complex networks. They propose a universal hierarchy measure based on Global Reaching Centrality and a visualization technique for any type of complex real-world network [22]. In a network, complexity often arises due to the interactions between similar units or as a result of nature of interactions (edges) and units (nodes). Hierarchy is an imperative feature of any complex network. However, the emergence of hierarchy within a network depends largely on the extent to which a node influences the other as well as the system in whole. Therefore, a node with the strongest influence can be regarded 'central' to a network. In other words, the nodes with a stronger impact can be at a higher order (rank) in a hierarchy. In fact, determining such nodes becomes crucial in defining a measure of hierarchy. Apart from a tree-like network, a real-world network is much more complicated with the existence of relationships between nodes of the same level, cycles of connected nodes, clusters, edges moving upwards etc. Hence hierarchy detection in networks is very demanding. Hierarchical measures so far been suggested cannot be applied on different complex systems due to many shortcomings. In order to define a measure based on reaching centralities, the paper essentially focus on flow hierarchies in real and adjustable hierarchical $(\mathrm{AH})$ networks.

The concept of Global Reaching Centrality (GRC) [21] measures the heterogeneous distribution of local reaching centralities in a graph. Local reaching centrality is largely based on a generalized case of $\mathrm{m}$-reach centrality with $\mathrm{m}=$ $N$; where $N$ is the no: of nodes in a given graph. In an unweighted directed graph, local reaching centrality is the ratio of number of nodes with finite positive directed distance from a particular node to the maximum number of reachable nodes from the same node. Therefore, the GRC of an unweighted directed graph can be defined as the difference between the highest and the average local reaching centralities within a network, given by [21]:

$$
\mathrm{GRC}=\frac{\sum_{i \in V}\left[C_{R}^{\max }-C_{R}(i)\right]}{N-1}
$$

$V=$ set of nodes; $C_{R}(i)=$ local reaching centrality; $C_{R}^{\max }=$ highest local reaching centrality; $N-1=$ maximum traversals possible.

For weighted undirected graphs, the generalization of GRC is quite straightforward based on local reaching centrality as defined for unweighted direct graphs. In the case of weighted directed graphs, the sum of lengths of all outgoing directed paths from node ' $i$ to node ' $j$ as well as the weight of edge along the path is taken into account. If there exist more than one directed shortest path from $i$ to $j$, then the path with maximum weight (i.e. maximum connection strength) is considered. Similarly, for an undirected unweighted graph, GRC can be obtained by excluding computation of weights of shortest path between two nodes. Further, GRC is observed on an adjustable hierarchy (AH) model. In an AH model, all nodes in a directed tree is assigned to a level 'l' such that the level of the root node is equal to the total number of levels and those at the bottom level has $I=1$. If a node has a level $l$, then the level of the child nodes would be 
$1-1$. Thereafter an additional no: of random edges are included in the model in such a way that $1-p$ proportion of edges is totally random. That is, two nodes chosen, say $\mathrm{A}$ and $\mathrm{B}$, are connected if they were not already connected in the $(\mathrm{AB})$ direction. The p proportion of the edges are connected as $(\mathrm{AB})$ only if, to preserve hierarchy. Randomization of real networks is done by generating a random network with the same in and out degree with respect to the original model and followed by choosing two random edges $\mathrm{AB}$ and $\mathrm{CD}$ and then changing the endpoints to obtain $\mathrm{AD}$ and $\mathrm{CB}$.

Analysis on a few classical networks such as Erdős-Rényi (ER) graphs [23], Scale-Free (SF) graphs and directed trees reveal that the GRC values are more acceptable than standard deviations of local reaching centralities to measure the hierarchical properties. The GRC for an adjustable hierarchical (AH) network is found to change continuously and monotonously in an interval of a highly random state to a fully hierarchical one. In the case of real networks, the edges are directed so that the origin of the edge has a greater impact on terminal. It has been observed that GRC depends largely on the average degree and network structure. A network with higher average degree has a smaller GRC indicating the existence of a lower hierarchy. However, the comparison of the actual GRC value with GRC of the randomized versions of the original networks exhibits slight variations. In order to analyze the correlation between hierarchy and controllability of a network, GRC is then compared with the number of driver nodes under switch board dynamics. Here, driver nodes are nodes that control the state of every edge. For a total control over an easily controllable network, the no: of driver nodes to be controlled are few. The results so obtained tend to exhibit a negative correlation between the two quantities, i.e. GRC and are inversely proportional to each other. This clearly suggests that a hierarchical network is better controllable.

The proposed hierarchical visualization technique for large graphs assigns each node into different levels on the basis of a local quantity. For an unweighted digraph this local quantity is equal to the local reaching centrality. Therefore, an ER graph posses a two layered hierarchical structure and arborescence has many layers. The structure of an SF graph lies in between an ER graph and an arborescence with a few clearly separated layers. To avoid different hierarchical lay-outs for single graphs of same graph model, ensembles of ER, SF, directed $\mathrm{AH}$ and real networks are visualized. In short, the proposed hierarchy measure, GRC quantifies the heterogeneity of local reaching centrality in whole network by introducing bidirectional edges among equivalent nodes. It is free from the drawbacks of the hierarchy measures so far been suggested. Hence, it can be concluded that GRC is a more suitable measure for hierarchy in any network.

\section{Classification of Networks}

Networks could be of different types. Some of them include: 
1) Physical networks comprising of physical entities and their interactions. Examples could be road network, world-maritime network etc., where cities/ ports are nodes and their routes are links,

2) Biological networks like protein-interaction networks, gene-regulatory network where proteins/genes form the nodes and their interactions form links,

3) Social networks where people or other entities become the nodes depending on the social context and their interactions being links.

Social network, where people represent nodes and the relationship between them represents links. A Link can be either directed (e.g. twitter where relationship is directional) or undirected (e.g. Facebook where relationship is mutual). Physical networks like Road network, World maritime network. Here nodes are represented by Cities and Ports respectively, and links are represented by routes. Biological networks: One of the examples is Protein-Protein interaction network where each protein is a node and interaction between them is represented by a link.

Among all the networks our focus is mainly on social networks. It shows some different properties in compare to other networks present like internet, World Wide Web. A network can be categorized into Infrastructure network and Interaction network. In infrastructure networks a link can be established even if the nodes do not interact with each other. Typical examples of infrastructure networks are Facebook, Orkut, Twitter, and etc. In Interaction network a link is setup among nodes when they interact with each other. Typical examples are Protein-Protein interaction network, Slashdot social network which is a result of interaction between nodes due to the threaded discussion. One major difference between the two networks is a link may lose its importance during the course of time in Infrastructure network i.e. a link might languish (or in other words stay static). For example, if one does not interact with a person on a regular basis then the link which connects both of them loses its importance with time. But in interaction network a link never loses its importance with time, as the nodes continue to interact with each other regularly.

A network can also be classified as signed and unsigned networks. In Unsigned networks, link between the nodes doesn't say about the nature of the link. Online social networks like Facebook, twitter, friendster, and etc come under this category. As opposed to unsigned network, in signed network a link carries +1 sign which represents a positive relationship or -1 sign which represents negative relationship among nodes. Both signs can be interpreted differently in different networks. For example in Eopinions network, +1 represents Trust while -1 represents Distrust, while in Slashdot Zoo network +1 represents friendship and -1 represents Foe ship between people.

\section{Hierarchy in Signed Networks}

Studies so far reveal only certain typical statistical properties shared by most of the complex networks. Some of distinctive properties include small-world phe- 
nomena [24], power-law degree distributions [25], clustering also called as network transitivity [26], community detection [22] etc. However, there still remain certain issues that are open. Hierarchy being one such issue has attracted many scientists.

Connectedness is a property exhibited by all networks and it determines the arrangement of nodes within a network. Such an arrangement gives rise to different classes of nodes based on certain factors that serve as a measure. In online social networks, actors tend to connect with each other within and across different classes on the basis of their perceived social hierarchy. The concept of social hierarchy can be stated as the emergence of a tree-like structure comprising of actors in a top-down fashion in the order of their ranks, describing a specific parent-child relationship. The total prestige owned by an actor could be considered as a measure of status. Therefore, a social hierarchy conveys a structure of authority and could be latent in every social network and needs to be extracted. Different literatures present a variety of approaches and measures for mining hierarchy in complex networks. Attempts have also been made to mine hierarchy in social networks. These are further discussed in the related literature section. However, in signed networks the hierarchy is far less discernible. The presence of negative interactions in signed networks, pose an additional challenge in deriving trust hierarchies from signed networks. Hence, we argue that the traditional notions are insufficient to derive hierarchies underlying signed networks.

In order to extract hierarchies from signed networks, we have considered the Slashdot and Epinions networks [27]. Slashdot is a technology related website, which has a feature named "Zoo" through which each user connects to other user as friend or foe based on the comments in a threaded discussion on an article. In the dataset, a friend is represented by directed edge of weight +1 and foe by directed edge of weight -1 . Epinions is a consumer review site where members of the network could decide whether to "trust" each other or not.

In this work, we attempt to mine hierarchies that remain latent in a signed network that represents the trust of nodes from the bottom to the root. It also based on a node's immediate neighborhood of trust relationships. Therefore, the trust hierarchy shows the nature of nodes trusting each other and at the same time preserves the locality of trust. These hierarchies are termed as locality-preserving trust hierarchies.

Being highly dynamic in nature, social networks have always reflected interesting patterns of connections among the nodes. These connections mostly lead to a parent-child relationship forming hierarchies among themselves. The hierarchical structure of a population in a social network often shapes the nature of the social interactions of individuals and, thus, provides insights into the underlying structure of the network. Understanding the mechanism by which hierarchies evolve is a fundamental question that still remains vague. Our approach could be relevant to a number of interesting current applications of social net- 
works including information dissemination, community structure detection and a framework for local self-governance among the population. The crux of our work lies in the fact that we seek to mine hierarchies based on the trust locality of a node in a signed network. That is, the hierarchies should be an abstract portrayal of local community structure.

\section{Interpretations of Hierarchies in Signed Network}

As discussed earlier, owing to the presence of positive (trust) as well as negative (distrust) interactions in signed networks (trust networks), the traditional notions of hierarchy were found to be inadequate to derive trust hierarchies. With the purpose of modeling both these interactions effectively into a hierarchy, we introduce two interpretations of trust or goodness into the trust networks. Trust is represented in terms of two different aspects namely, presence of trust and absence of distrust. In fact, these two interpretations could be considered as duals of trust signifying the degree of goodness of an actor. Presence of trust would imply how good an actor is where as an absence of distrust would imply how less bad the actor actually is. Consequently, the trust-based hierarchies thus obtained would consist of several actors arranged in the order of their degree of trust. This could be illustrated in Figure 3, as follows:

Figure 3 and Figure 4, illustrates the trust-based hierarchies existing among actors. It is to be noted that in a trust network, high distrust and low trust need not necessarily be the same. In Figure 3, the trust earned is high at the root node indicating high goodness and decreases gradually as we move down the hierarchy. That is, the actors at the bottom of the hierarchy would have a comparatively lower trust than those at the top. On the same note, in Figure 4, the absence of distrust is found to decrease as one moves down the hierarchy beginning at the root. That is, at the root node the absence of distrust is much higher

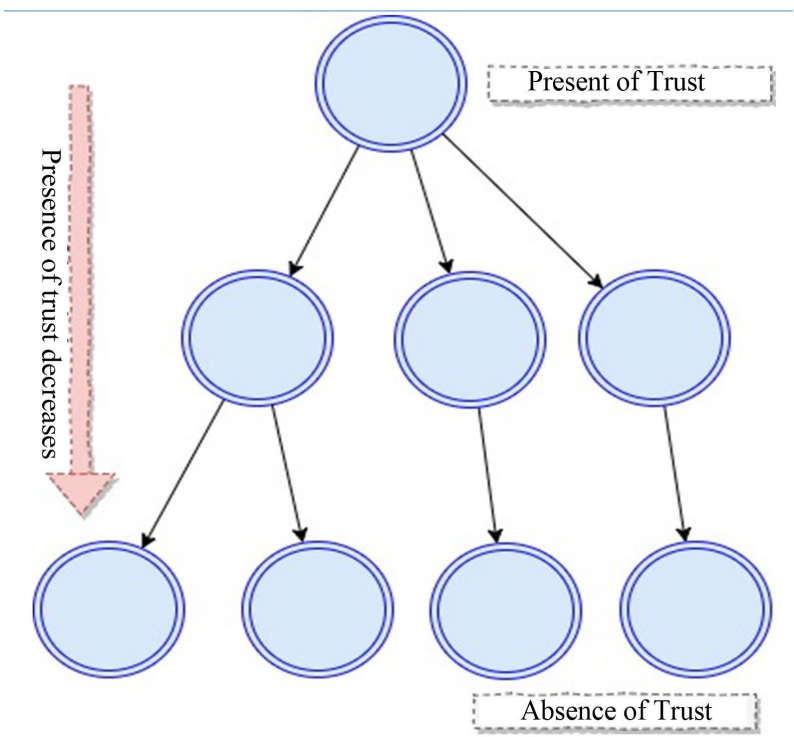

Figure 3. Hierarchy based on the presence of trust. 


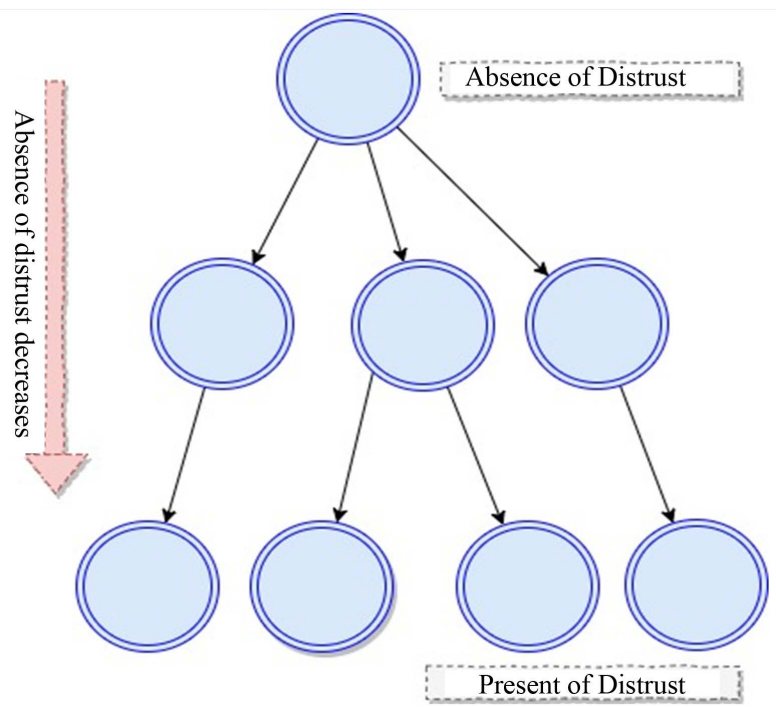

Figure 4. Hierarchy based on Absence of distrust.

in turn indicating low badness when compared to those at the bottom of the hierarchy.

Alternatively, it could be viewed that 'goodness' decreases as we move down a trust hierarchy and 'badness' increases as we move down the distrust hierarchy. This view puts forward a question of the manner in which an actor is considered to be genuinely good or bad. The philosophy behind this view could be explained in terms of the collective opinion of the population. Social networks comprise of autonomous agents capable of expressing opinions on their own. These opinions solely are based on their independent cognitive processes or inferences. In other words, the opinion of an actor is not hampered by any party or an interest group in particular. Therefore, a collective opinion regarding the trustworthiness of an actor cannot be ruled out as a co-incidence. With time, an architecture entirely based on trust emerges. This emergent trust-based architecture eventually becomes acceptable to the whole population. Thus an actor who has earned the trust (distrust) through the unanimous opinion of the majority is considered to be genuinely trustworthy (untrustworthy). By means of this emergent architecture it is possible to gain new insights into patterns underlying a network. An interesting example in this regard could be the collaborative editing of content in Wikipedia pages. Readers are allowed to edit information related to a particular topic and over time, an information architecture evolves eventually reaching consensus among the editors.

However, so as to convey both trust and distrust effectively in a single hierarchy, the trust as well as the distrust earned by an actor are taken together in terms of their aggregate deserve values. That is, deserve of node $u$ is the aggregate of the trust and distrust it earns from its neighbors. The trust/distrust from node $\mathrm{v}$ to node $\mathrm{u}$ is dampened based on its bias towards trusting or distrusting the population at large. Therefore, the higher the bias, the lower is the effect of v's vote to $u$. Thus a consolidated hierarchy of actors is formed by way of a par- 
ent-child relationship, viz. child trusts parent. Here, the actors are arranged into different levels according to the deserve values within their neighborhood of trust. That is, the consolidated hierarchy not only portrays the aggregate trust earned but also preserves the locality of trust of an actor. Therefore, the hierarchy thus obtained is said to represent a locality-based social structure in the descending order of aggregate deserves.

\section{Conclusions and Future Work}

The primary objective of our work was to mine locality-preserving trust hierarchies from signed networks. We discussed the different approaches adopted to mine hierarchies in complex networks in various fields ranging from sociology, biology to computer science. In addition to this, we explained why, unlike other networks, mining hierarchies in signed networks is novel. In trust-based networks, there is an explicit show of trust and distrust, very similar to real-world interactions. As a result, a trust-architecture evolves giving rise to an underlying social hierarchy. Then, we proposed the two interpretations associated with trust in signed networks and observed the nature of hierarchies derived from the above assumptions.

A future line of work could be a method for merging of hierarchies in order to arrive at the desired(smaller) number of hierarchies, without compromising on the locality-preservation to examine the patterns underlying it. Implementing other hypotheses relevant to locality-preserving hierarchy construction based on the application context. On a similar note, we plan to extend our approach and also analyze other signed networks such as Essembly etc.

\section{References}

[1] Borgatti, S.P., Mehra, A., Brass, D.J. and Labianca, G. (2009) Network Analysis in the Social Sciences. Science, 323, 892-895.

[2] Sparrow, M.K. (1991) The Application of Network Analysis to Criminal Intelligence: An Assessment of the Prospects. Social Networks, 13, 251-274. https://doi.org/10.1016/0378-8733(91)90008-H

[3] Hodder, I. and Mol, A. (2016) Network Analysis and Entanglement. Journal of Archaeological Method and Theory, 23, 1066-1094. https://doi.org/10.1007/s10816-015-9259-6

[4] Wang, J., Zuo, X. and He, Y. (2010) Graph-Based Network Analysis of Resting-State Functional MRI. Frontiers in Systems Neuroscience, 4, 4-16. https://doi.org/10.3389/fnsys.2010.00016

[5] Jørgensen, S.E., Patten, B.C. and Straškraba, M. (1992) Ecosystems Emerging: Toward an Ecology of Complex Systems in a Complex Future. Ecological Modelling, 62, 1-27. https://doi.org/10.1016/0304-3800(92)90080-X

[6] O’Malley, A.J. and Onnela, J.P. (2014) Topics in Social Network Analysis and Network Science. arXiv preprint arXiv:1404.0067.

[7] Kaur, H. and He, J.S. (2017) Blocking Negative Influential Node Set in Social Networks: From Host Perspective. Transactions on Emerging Telecommunications Technologies, 28, 1-62. https://doi.org/10.1002/ett.3007 
[8] Li, Y., Chen, W., Wang, Y. and Zhang, Z.L. (2013) Influence Diffusion Dynamics and Influence Maximization in Social Networks with Friend and Foe Relationships. Proceedings of the Sixth ACM International Conference on Web Search and Data Mining, Rome, Italy, 4-8 February 2013, 657-666. https://doi.org/10.1145/2433396.2433478

[9] Kunegis, J. (2013) Konect: The Koblenz Network Collection. Proceedings of the 22nd International Conference on World Wide Web, Rio de Janeiro, Brazil, 13-17 May 2013, 1343-1350. https://doi.org/10.1145/2487788.2488173

[10] Bonabeau, E., Theraulaz, G. and Deneubourg, J.L. (1999) Dominance Orders in Animal Societies: The Self-Organization Hypothesis Revisited. Bulletin of mathematical biology, 61, 727-757. https://doi.org/10.1006/bulm.1999.0108

[11] Nagy, M., Akos, Z., Biro, D. and Vicsek, T. (2010) Hierarchical Group Dynamics in Pigeon Flocks.

[12] Leblebici, H. and Whetten, D.A. (1984) The Concept of Horizontal Hierarchy and the Organization of Interorganizational Networks: A Comparative Analysis. Social Networks, 6, 31-58.

[13] Ravasz, E. and Barabási, A.L. (2003) Hierarchical Organization in Complex Networks. Physical Review E, 67, Article ID: 026112. https://doi.org/10.1103/PhysRevE.67.026112

[14] Dorogovtsev, S.N., Goltsev, A.V. and Mendes, J.F.F. (2002) Pseudofractal Scale-Free Web. Physical Review E, 65, Article ID: 066122. https://doi.org/10.1103/PhysRevE.65.066122

[15] Rowe, R., Creamer, G., Hershkop, S. and Stolfo, S.J. (2007) Automated Social Hierarchy Detection through Email Network Analysis. In: Proceedings of the 9th WebKDD and 1st SNA-KDD 2007 Workshop on Web Mining and Social Network Analysis, ACM, New York, 109-117. https://doi.org/10.1145/1348549.1348562

[16] Liben-Nowell, D. and Kleinberg, J. (2007) The Link-Prediction Problem for Social Networks. Journal of the Association for Information Science and Technology, 58, 1019-1031. https://doi.org/10.1002/asi.20591

[17] Gilbert, E. and Karahalios, K. (2009) Predicting Tie Strength with Social Media. In: Proceedings of the SIGCHI Conference on Human Factors in Computing Systems, ACM, New York, 211-220.

[18] Helic, D. and Strohmaier, M. (2011) Building Directories for Social Tagging Systems. In: Proceedings of the 20th ACM International Conference on Information and Knowledge Management, ACM, New York, 525-534. https://doi.org/10.1145/2063576.2063655

[19] Maiya, A.S. and Berger-Wolf, T.Y. (2009) Inferring the Maximum Likelihood Hierarchy in Social Networks. International Conference on Computational Science and Engineering, Vancouver, 29-31 August 2009, Vol. 4, 245-250.

[20] Gupte, M., Shankar, P., Li, J., Muthukrishnan, S. and Iftode, L. (2011) Finding Hierarchy in Directed Online Social Networks. In: Proceedings of the 20 th International Conference on World Wide Web, ACM, New York, 557-566. https://doi.org/10.1145/1963405.1963484

[21] Mones, E., Vicsek, L. and Vicsek, T. (2012) Hierarchy Measure for Complex Networks. PLoS ONE, 7, e33799. https://doi.org/10.1371/journal.pone.0033799

[22] Girvan, M. and Newman, M.E. (2002) Community Structure in Social and Biological Networks. Proceedings of the National Academy of Sciences, 99, 7821-7826.

https://doi.org/10.1073/pnas.122653799 
[23] Seshadhri, C., Kolda, T.G. and Pinar, A. (2012) Community Structure and Scale-Free Collections of Erdős-Rényi Graphs. Physical Review E, 85, Article ID: 056109. https://doi.org/10.1103/PhysRevE.85.056109

[24] Travers, J. and Milgram, S. (1967) The Small World Problem. Phychology Today, 1, 61-67.

[25] Barabási, A.L. and Albert, R. (1999) Emergence of Scaling in Random Networks. Science, 286, 509-512.

[26] Watts, D.J. and Strogatz, S.H. (1998) Collective Dynamics of "Small-World" Networks. Nature, 393, 440. https://doi.org/10.1038/30918

[27] Leskovec, J., Huttenlocher, D. and Kleinberg, J. (2010) Signed Networks in Social Media. In: Proceedings of the SIGCHI Conference on Human Factors in Computing Systems, ACM, New York, 1361-1370. 